\title{
Gradhiva
}

GRADHI

Revue d'anthropologie et d'histoire des arts

Autour de Lucien Sebag

\section{Les archives photographiques du Laboratoire d'anthropologie sociale}

Anne-Laure Pierre

\section{(2) OpenEdition}

\section{Journals}

Édition électronique

URL : http://journals.openedition.org/gradhiva/519

DOI : 10.4000/gradhiva. 519

ISSN : 1760-849X

Éditeur

Musée du quai Branly Jacques Chirac

\section{Édition imprimée}

Date de publication : 1 novembre 2005

Pagination : 147-148

ISBN : 2-915-133-10-7

ISSN : 0764-8928

Référence électronique

Anne-Laure Pierre, "Les archives photographiques du Laboratoire d'anthropologie sociale », Gradhiva [En ligne], 2 | 2005, mis en ligne le 10 décembre 2008, consulté le 15 septembre 2020. URL : http:// journals.openedition.org/gradhiva/519

Ce document a été généré automatiquement le 15 septembre 2020

(c) musée du quai Branly 


\title{
Les archives photographiques du Laboratoire d'anthropologie sociale
}

\author{
Anne-Laure Pierre
}

Depuis octobre 2004, la bibliothèque du Laboratoire d'Anthropologie Sociale (LAS) a entrepris un inventaire informatisé des photographies conservées depuis 1960 dans son fonds d'archives. Le fonds restait en attente depuis sa création, les photographies ayant simplement fait l'objet d'un enregistrement sur des cahiers manuscrits, avec des disparités dans l'état de conservation des photographies. Une partie était bien conservée : les épreuves et les films noir et blanc rangés sous enveloppe, dans une armoire close, sont restés intacts. En revanche, une autre partie, les diapositives couleurs, souffrait d'un stockage inadapté. La fragilité des photographies couleurs, dont la durée de vie est courte, demande des mesures de conservation préventive strictes. La qualité chromatique de certaines photographies était très dégradée, ce qui les rendait inconsultables.

\section{Spécificité des documents photographiques}

D’une manière générale, l'intérêt historique des photographies détenues par le Laboratoire restait inconnu de la communauté scientifique, et ceci pour une raison simple : il était difficile d'y avoir accès. Alors que, depuis quelques années, les archives manuscrites des chercheurs commençaient d'être inventoriées, les photographies restaient toujours à l'écart, étant suspectées, à juste titre, de poser des problèmes spécifiques tant au point de vue de leur traitement physique que documentaire.

3 La mauvaise interprétation des qualités d'un document peut provoquer des erreurs préjudiciables à sa perception même. Les photographies originales déposées par les anthropologues à la suite de missions représentent un matériau hétérogène - sur des supports différents et dans des formats divers - dont la signification n'est pas livrée avec l'objet, comme l'imprimé, et dont on ne situe pas très bien les limites de l'analyse. La conscience du caractère polysémique de la photographie fait que la réponse la plus courante est d'assigner l'image à son usage documentaire dans la discipline, par 
exemple, en anthropologie, de limiter l'indexation à l'ethnie ou la géographie. Mais ce choix résulte essentiellement d'une méconnaissance de l'histoire de la photographie, et de sa place tant en sciences sociales qu'en histoire de l'art. Aujourd'hui, le traitement des collections photographiques doit permettre de les faire entrer dans un champ d'études plus large, notamment celui de l'anthropologie visuelle, ou celui des Visual studies, qui privilégie des approches comparatistes et historiques sur la photographie ${ }^{1}$.

Il est extrêmement important de situer une photographie dans son contexte de prise de vue, c'est-à-dire de la lier à un événement, un voyage, une mission, de tenter de dater le document le plus précisément possible pour le placer au plus près du moment où il est né. La force cognitive des photographies est une évidence. Soulignons simplement ceci : le sujet principal des photographies en ethnologie étant l'homme, ses activités, sa vie sociale, l'impact de sa représentation est très fort émotionnellement. Elle se fixe durablement dans les esprits et charge l'image de représenter une réalité. C'est pourquoi la connaissance la plus exacte possible de l'origine historique des images doit toujours équilibrer leur vision.

\section{Politique documentaire}

5 Cette orientation que nous avons prise était facilitée par le traitement archivistique choisi précédemment par la direction de la bibliothèque. En effet, le standard de description des archives ISAD- $\mathrm{G}^{2}$, norme appliquée au LAS, permet de mettre en avant les notions de contexte de création, la description matérielle de l'objet, et donc de sortir du schéma image-cote-indexation par mots-clefs, qui est celui des banques d'images ou des photothèques, modèles que nous voulions rejeter.

6 La numérisation des photographies est un outil très performant pour les visualiser, ce qui est le but essentiellement recherché, mais cette possibilité d'avoir une reproduction virtuelle de la photographie rend la réflexion préalable d'autant plus importante. Trop d'images circulent sans être reliées à un contexte de production et le rôle d'une collection publique, a fortiori la collection d'un laboratoire scientifique, est d'avoir une approche déontologique forte en la matière, en mettant à disposition des chercheurs et des étudiants un outil à portée pédagogique.

7 Il faut donc restituer fidèlement, dans la mesure du possible en prenant en note ce qui figure sur le document lui-même, les notions d'auteur, d'événement, de date. Lorsqu'on ne dispose pas de ces informations, il est important de nommer dans la notice l'auteur du renseignement apporté et la date à laquelle il le donne. Il faut également respecter la description exacte du support matériel dans lequel l'image est fixée, ce qui suppose une connaissance historique du médium.

Définir s'il s'agit d'une plaque de verre, d'une photographie prise au moyen format (6x $6 \mathrm{~cm}$ ), d'un film noir et blanc $24 \times 36 \mathrm{~mm}$, ce n'est pas seulement dater le document d'après la technique utilisée le cas échéant, le conserver de manière adéquate (ce qui rend possible la gestion des collections), c'est aussi noter une information qui nous indique quelle compréhension avait le photographe de son outil. Enfin, lorsque c'est possible, il est très intéressant d'archiver un film photographique dans son intégralité, de manière à pouvoir suivre le regard du photographe image après image, dans un déroulement en séquence. Le respect de cette approche globale garantit la signification historique des photographies. 
Ces critères d'analyse étant posés, les soucis récurrents sur le droit à l'image et le droit d'auteur sont au moins limités, dans la mesure où la responsabilité de l'auteur est identifiée, mais aussi la responsabilité du médiateur de la collection, en l'occurrence la bibliothèque. La diffusion des photographies est en effet restreinte à un cadre de recherche et sera toujours fortement liée à ce qui a été défini : la primauté du contexte de production de l'archive ${ }^{3}$.

\section{Premiers résultats}

10 Depuis octobre 2004, 5600 photographies du LAS sont déjà sorties de l'ombre, et c'est un grand bonheur de constater encore une fois la richesse infinie de l'archive. Les photographies de Lucien Sebag, utilisées dans ce dossier, certaines photographies d'Alfred Métraux publiées dans le numéro précédent de Gradhiva résultent de ce travail. Mais l'intérêt ne se limite pas à la constitution d'un fonds d'illustration, fonction certes importante. Ce qui rend le travail passionnant, c'est de bâtir une collection structurée par des lignes historiques, qui dessinent l'identité du lieu de réception et de la discipline en question, et forment une généalogie.

Le fonds débute avec un des pères de la discipline, Robert Hertz, se poursuit avec Alfred Métraux, José Maria Cruxent (mission franco-vénézuelienne du haut Orénoque, 1950); une deuxième génération commence avec Lucien Sebag et Jacques Lizot. Dans le fonds des photographies de la mission Minot, l'ancêtre est Hippolyte Amiot, menuisier et photographe à Minot vers 1900, dont les membres de la mission sauvent les images promises à la destruction et feront une large utilisation ethnographique et illustrative, les confrontant à des images de 1970 et 1980 prises au moment de l'enquête.

Cette appréciation des photographies comme support d'un lien social trouve un autre prolongement dans la volonté des anthropologues de revenir sur le terrain avec leurs images, pour les montrer aux plus jeunes générations. Ainsi au mois de mai dernier, Carlos Valiente Noailles est reparti au Botswana avec des épreuves sur papier pour, ditil, "finir son livre», comme si les photographies allaient lui permettre de boucler l'histoire. Mais il y aurait bien d'autres exemples de cette démarche.

13 D'autres histoires de parenté donnent des pistes sur la production des photographies et la façon dont les anthropologues s'y sont préparés grâce aux liens avec des photographes professionnels. La relation d'Alfred Métraux avec Pierre Verger, dont la collection détient un témoignage important, les photographies et le récit du voyage qu'ils firent ensemble au Bénin (alors Dahomey) en 1952-1953 en est l'exemple le plus frappant. Un autre exemple : Jacques Lizot demande des conseils pour la prise de vue à Théa, photographe roumaine ayant participé à l'expédition du haut Orénoque (et dont quelques photographies figurent par ailleurs dans le fonds Cruxent). Il écrit : «Théa doit m'aider à faire de meilleures photographies » (cahier de terrain $\mathrm{n}^{\circ} 21$, octobre 1969, fonds Jacques Lizot).

14 Les photographies ne sont donc pas prises par hasard, elles ne résultent pas d'une approche velléitaire, mais d'un désir profond d'enregistrer un fragment de la réalité du terrain et de confronter ces matériaux non verbaux à l'écrit. L'emploi du moyen format chez Lucien Sebag (comme chez Métraux), indique qu'il ne s'agit pas de faire des images en passant, mais bien de se placer en face de son sujet, la plupart des personnes prises en portrait arborant un regard consentant. À travers leur représentation, ils 
participent activement à l'enquête. Le cas du fonds Sebag est exemplaire dans le cadre du projet de valorisation que nous menons, car on aurait pu croire qu'il était vain de prendre en charge cette collection, tant elle paraissait altérée. L'émulsion des diapositives (ektachrome) a mal résisté au temps, mais heureusement la numérisation rend possible le recalcul des couleurs d'origine et permet de restituer ces photographies au regard.

Le Laboratoire d'Anthropologie Sociale révèle, avec ces archives, un nouvel aspect de l'histoire des usages de la photographie comme matériau de terrain : nouveau par la personnalité de ses membres, nouveau par l'époque couverte par les documents, particulièrement les années 1960-1970, nouveau par les terrains, avec une prédilection pour l'Amérique du Sud. Les photographies des archives du LAS, prolongent ainsi une histoire commencée ailleurs. Prochainement, si le programme d'inventaire peut se poursuivre, de nouvelles photographies enrichiront cette réflexion.

\section{NOTES}

1. Consulter le site internet de Lhivic (Laboratoire d'histoire visuelle contemporaine), et plus particulièrement le résumé d'une conférence de Maren Stange, Visual culture and visual studies in the United States (www.lhivic.org/seminaires/lci8.html ; consulté le 5 juillet 2005).

2. International Standard Archival Description: norme de description archivistique qui permet une description des fonds à plusieurs niveaux, allant du général au particulier.

3. La consultation des photographies au Laboratoire d'Anthropologie Sociale est soumise à une demande motivée dans le cadre d'une recherche scientifique ou à des fins illustratives pédagogiques.

\section{AUTEUR}

\section{ANNE-LAURE PIERRE}

annelaure.pierre@free.fr 